\title{
PET imaging with copper-64 as a tool for real-time in vivo investigations of the necessity for crosslinking of polymeric micelles in nanomedicine
}

Imaging the influence of polymeric micelle crosslinking

Jensen, Andreas Tue Ingemann; Binderup, Tina; Ek, Pramod Kumar; Grandjean, Constance E; Rasmussen, Palle; Kjær, Andreas ; Andresen, Thomas Lars

Published in:

Journal of Labelled Compounds and Radiopharmaceuticals

Link to article, DOI:

$10.1002 /$ jlcr.3510

Publication date:

2017

Document Version

Peer reviewed version

Link back to DTU Orbit

Citation (APA):

Jensen, A. T. I., Binderup, T., Ek, P. K., Grandjean, C. E., Rasmussen, P., Kjær, A., \& Andresen, T. L. (2017). PET imaging with copper-64 as a tool for real-time in vivo investigations of the necessity for crosslinking of polymeric micelles in nanomedicine: Imaging the influence of polymeric micelle crosslinking. Journal of Labelled Compounds and Radiopharmaceuticals, 60(8), 366-374. https://doi.org/10.1002/jlcr.3510

\section{General rights}

Copyright and moral rights for the publications made accessible in the public portal are retained by the authors and/or other copyright owners and it is a condition of accessing publications that users recognise and abide by the legal requirements associated with these rights.

- Users may download and print one copy of any publication from the public portal for the purpose of private study or research.

- You may not further distribute the material or use it for any profit-making activity or commercial gain

- You may freely distribute the URL identifying the publication in the public portal 


\section{PET imaging with copper-64 as a tool for real-time}

in vivo investigations of the necessity for crosslinking of polymeric micelles in

\section{nanomedicine}

Andreas I. Jensen ${ }^{1 *}$, Tina Binderup ${ }^{2}$, Pramod Kumar EK ${ }^{3}$, Constance E. Grandjean ${ }^{2}$, Palle H. Rasmussen $^{1}$, Andreas Kjær ${ }^{2}$, Thomas L. Andresen ${ }^{3 *}$

1. Technical University of Denmark, DTU Nutech, Center for Nanomedicine and Theranostics, 4000 Roskilde, Denmark

2. Rigshospitalet and University of Copenhagen, Dept. of Clinical Physiology, Nuclear Medicine \& PET and Cluster for Molecular Imaging, 2100 Copenhagen, Denmark

3. Technical University of Denmark, DTU Nanotech, Center for Nanomedicine and Theranostics, 2800 Lyngby, Denmark

Address correspondence to: A.I. Jensen: atije@dtu.dk, +45 20126187 or T.L. Andresen: tlan@nanotech.dtu.dk, +45 25374486.

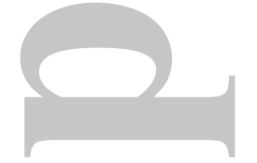

KEYWORDS: Polymeric micelles, Copper-64, PET, crosslinking, CB-TE2A, nanomedicine

This article has been accepted for publication and undergone full peer review but has not been through the copyediting, typesetting, pagination and proofreading process which may lead to differences between this version and the Version of Record. Please cite this article as doi: $10.1002 / j$ lcr.3510 


\section{ABSTRACT}

Polymeric micelles in nanomedicine are often crosslinked to prevent disintegration in vivo. This typically requires clinically problematic chemicals or laborious procedures. In addition, crosslinking may interfere with advanced release strategies. Despite this, it is often not investigated whether crosslinking is necessary for efficient drug delivery. We used PET imaging with ${ }^{64} \mathrm{Cu}$ to demonstrate general methodology for real-time in vivo investigations of micelle stability. Triblock copolymers with 4-methylcoumarin cores of ABC-type (PEGPHEMA-PCMA) were functionalized in the handle region (PHEMA) with CB-TE2A chelators. Polymeric micelles were formed by dialysis and one half was core-crosslinked by UV light (CL) and the other half was not (nonCL). Both CL and nonCL were radiolabeled with ${ }^{64} \mathrm{Cu}$ and compared in vivo in tumor-bearing mice, with free ${ }^{64} \mathrm{Cu}$ as control. Accumulation in relevant organs was quantified by ROI analysis on PET images and ex vivo counting. It was observed that $\mathrm{CL}$ and nonCL showed limited differences in biodistribution from each other, whereas both differed markedly from control (free ${ }^{64} \mathrm{Cu}$ ). This demonstrated that 4-methylcoumarin core micelles may form micelles that are stable in circulation even without crosslinking. The methodology presented here where individual unimers are radiolabeled is applicable to a wide range of polymeric micelle types.

\section{Introduction}

Polymeric micelles (PMs) are nanoparticles composed of linear, synthetic monomers that contain several chemically distinct domains known as blocks. ${ }^{1}$ Usually di- or triblock micelles are prepared, where the triblock variety may contain 1) a hydrophobic domain making up the core, 2) a handle domain that can be chemically functionalized, and 3) a hydrophilic domain that faces the surrounding aqueous medium (Figure 1). Such monomers can self-assemble into nanostructures that can be engineered to exhibit a wide range of sizes (usually $5-100 \mathrm{~nm}$ ) and to be highly synthetically versatile. Polymeric micelles can accumulate in tumors through the enhanced permeation and retention (EPR) effect ${ }^{2}$ and have potential as drug delivery systems, in particular by covalently binding drug molecules through labile bonds that can break at the desired site of action., 
The stability of polymeric micelles is in part determined by the critical micelle concentration (CMC). This number denotes the maximum concentration at thermodynamic equilibrium at which single monomers can exist freely in aqueous solution, and hence at which point they will start to form micelles. In circulation, polymeric micelles may disintegrate due to dilution below the $\mathrm{CMC}$, as well as by interactions with blood-born proteins that shift the equilibrium towards the unimer state. ${ }^{1}$ This causes possible release of drug molecules and the abrogation of the desirable nanoparticulate properties of the micelles. In order to circumvent this, the monomers of polymeric micelles have been crosslinked by a multitude of methods, in order to create an integral nanoparticle. These have included amide formation, ${ }^{5}$ polymerization reactions $^{6,7}$, UV-induced initiator-mediated photo-crosslinking ${ }^{8,9}$ as well as more exotic methods. ${ }^{10,11}$ Reversible crosslinking strategies have been devised as a manner in which to control the release of cargo drug molecules, once accumulation at target sites has been achieved. This has been achieved using disulfide bonds, ${ }^{12}$ cleavable imine linkages ${ }^{13}$ and direct dimerization of core coumarin moieties through irradiation with ultraviolet light. ${ }^{14}$ This latter strategy is known as photo core crosslinking (PCCL) and has the immediate advantage that no addition of reactive chemicals, initiators or purification steps are necessary. Coumarin undergoes $2+2$ photodimerization to a cyclobutane ring when exposed to UV light of $\lambda>310$ $\mathrm{nm}$. If irradiated at $\lambda<260 \mathrm{~nm}$, the ring is cleaved, refurnishing the two coumarin moieties as separate molecules. The chemical properties of coumarin as a reversibly dimerizable compound were excellently reviewed by Trenor et al. ${ }^{15}$ and its applications by He et al. ${ }^{16}$ As the crosslinkable carbon-carbon double bond of coumarin absorbs light around $320 \mathrm{~nm}$, the degree of crosslinking can be assessed by measuring the drop in absorbance at this wavelength. As coumarin with a methyl group in the 4-position exhibits faster dimerization rates than other coumarin derivates ${ }^{17}$, 4-methyl coumarin is often employed in micelle crosslinking. ${ }^{14,18}$

When investigating crosslinking strategies, it is important to evaluate the stability of the resulting polymeric micelles against their non-crosslinked counterparts. As non-crosslinked PMs may have certain advantages, such as easier renal excretion and easier preparation, there is a rationale in not crosslinking unless the benefits are well-documented. A useful technique for investigating such key in vivo properties of nanoparticles is labeling with positronemitting radionuclides and imaging by positron emission tomography (PET). PET is based on the coincidence detection of positrons from positron-electron annihilation and is a truly quantitative technique which offers excellent sensitivity and spatial resolution. When imaging 
nanoparticles in murine models, an especially useful radionuclide is copper-64 $\left({ }^{64} \mathrm{Cu}, \mathrm{T}_{1 / 2}=\right.$ $12.7 \mathrm{~h}$ ), which offers an imaging window of up to 48-72 hours and thereby adequately covers the distribution phase of long-circulating PMs. Association of ${ }^{64} \mathrm{Cu}$ to nanoparticles is typically achieved by complexation in a covalently bound chelator molecule. The crossbridged, macrocyclic chelator 2,2'-(1,4,8,11-tetraazabicyclo[6.6.2] hexadecane-4,11diyl)diacetic acid (CB-TE2A) has been reported on several occasions to exhibit excellent serum and blood stream stability and is generally considered to be a highly reliable chelator for ${ }^{64} \mathrm{Cu} .{ }^{19,20,21}$

In the study at hand we employed in vivo PET imaging to investigate the difference in biodistribution between a crosslinked and a non-crosslinked version of two identical triblock coumarin-core polymeric micelles radiolabeled with ${ }^{64} \mathrm{Cu}$ through $\mathrm{CB}-\mathrm{TE} 2 \mathrm{~A}$. The CB-TE2A was conjugated to the middle block, where it would be shielded from interaction with in vivo components by the outermost polyethylene glycol (PEG)-layer, limiting the effects the chelate may have on the biodistribution of the micelles.

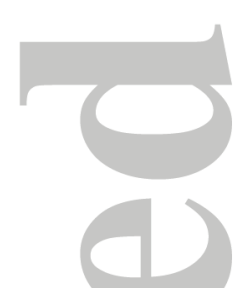

\section{Experimental}

\subsection{Materials}

CB-TE2A $2 \mathrm{H}_{2} \mathrm{O}$ was purchased from Macrocyclics. All further chemicals and solvents were from Sigma-Aldrich. For anhydrous syntheses, dimethylformamide (DMF) was dried over $4 \AA$ molecular sieves and all glassware was oven-dried overnight or heatgun-dried prior to use. Reactions were carried out under a nitrogen atmosphere and polymers and micelles containing coumarin moieties were shielded from light whenever possible. Cellulose membrane dialysis tubing (12.4 kDa cutoff) was from Sigma Aldrich. Size-exclusion chromatography (SEC) was carried out on custom-built automated equipment, using Sephadex G25 "fine" (GE Healthcare, fractionation range: 1000-5000 Da) as stationary phase and $10 \mathrm{mM}$ piperazine-N,N'-bis(2-ethanesulfonic acid) (PIPES) buffer (pH 7.0, $150 \mathrm{mM}$ $\mathrm{NaCl}$ ) as eluent, flow rate of $0.5 \mathrm{~mL} / \mathrm{min}$, room temperature and column dimensions of $20 \times$ $1.5 \mathrm{~cm}$. Photo cross-linking by UV irradiation was done on an Omnicure Series 1000 (Lumen 
Dynamics). UV-VIS spectra were recorded on a Unicam Helios Uni 4923 spectrophotometer. Size and zeta potential were measured on a Brookhaven ZetaPALS. ${ }^{64} \mathrm{Cu}$ was produced on a GE PETtrace Cyclotron. Radio-TLCs were analyzed on a Raytest MiniGita Star and eluted with $5 \%$ (w/v) $\mathrm{NH}_{4} \mathrm{OAc}$ in $\mathrm{H}_{2} \mathrm{O}-\mathrm{MeOH}(1: 1)$. Rf of ${ }^{64} \mathrm{Cu}$-EDTA: 0.7 (EDTA: ethylenediaminetetraacetic acid), Rf of ${ }^{64} \mathrm{Cu}-\mathrm{CB}-\mathrm{TE} 2 \mathrm{~A}: 0.3$.

\subsection{Preparation of CB-TE2A conjugated polymeric micelles}

The synthesis and micellization of CB-TE2A conjugated polyethylene glycolpolyhydroxyethyl methacrylate-polymethacryloyloxyethoxy-4-methylcoumarin (PEGPHEMA-PCMA) unimers was carried out as previously reported by isolated macroinitiator atomic transfer radical polymerization (ATRP) (Figure 1). ${ }^{22}$ In brief, the PEG-PHEMA-Cl block copolymer was synthesized according to a literature procedure ${ }^{23}$ and it was used as macroinitiator for the synthesisis of the 4-methylcoumarin block. The resulting triblock copolymer PEG-PHEMA-PCMA was precipitated from methanol and dried under vacuum. Copper residues and residual impurities were removed by dialysis (molecular weight cut-off $=12 \mathrm{kDa}$ ). CB-TE2A was conjugated by ester bonds to the primary alcohols of the PHEMA block through 4-dimethylaminopyridine (DMAP)-catalyzed 1-ethyl-3-(3dimethylaminopropyl)carbodiimide (EDC) coupling in DMF. The micelles were subsequently formed by dialysis with water.

\subsection{Crosslinking of micelles}

Micelle dispersions $(1100 \mu \mathrm{L})$ were transferred to a $4 \mathrm{~mL}$ glass vial which was placed in a water-ice bath kept at 5-7 ${ }^{\circ} \mathrm{C}$. Under vigorous magnetic stirring, the dispersions were irradiated with UV light for $2 \times 15$ minutes at an intensity of $2 \mathrm{~W} / \mathrm{cm}^{2}$ and a wavelength of $320<\lambda<500 \mathrm{~nm}$. The UV probe was placed above the water surface at a distance of about a centimeter. The degree of crosslinking (\%CL) was determined by measuring the drop in absorbance $(A)$ at $320 \mathrm{~nm}$ and calculating: $\left(\left(\mathrm{A}_{\text {before }}-\mathrm{A}_{\text {after }}\right) /\left(\mathrm{A}_{\text {before }}-\mathrm{A}_{0}\right)\right) \times 100 \%=\% \mathrm{CL}$, where $A_{0}$ was set to zero. ${ }^{24}$ 


\subsection{Radiolabeling}

To a vial containing dried ${ }^{64} \mathrm{CuCl}_{2}$ was added aqueous $\mathrm{NH}_{4} \mathrm{OAc}(100 \mu \mathrm{L}, 0.1 \mathrm{M}, \mathrm{pH} 5.5)$, followed by magnetic stirring at room temperature for 10 minutes. Then was added crosslinked (CL) or non-crosslinked (nonCL) PM dispersions (400 $\mu \mathrm{L}, \sim 4 \mathrm{mg} / \mathrm{mL}$ ) and the mixtures were stirred for 3 hours at $80{ }^{\circ} \mathrm{C}$. After radiolabeling, non-incorporated ${ }^{64} \mathrm{Cu}$ was scavenged by immediate addition of aqueous EDTA $(50 \mu \mathrm{L}, 1 \mathrm{mM})$ followed by stirring for 20 minutes. The radiolabeled PMs were then separated from ${ }^{64} \mathrm{Cu}$-EDTA by automated sizeexclusion chromatography using Sephadex 25 as stationary phase and isotonic PIPES buffer (10 mM PIPES, $150 \mathrm{mM} \mathrm{NaCl}, \mathrm{pH}$ 7.0) as eluent. From the fractions making up the largemolecules peak (containing the PMs) a total volume of $1 \mathrm{~mL}$ which contained the highest concentration of radioactivity was collected and used for in vivo studies.

\subsection{Characterization of micelles}

Micelles were characterized by dynamic light scattering (DLS), providing hydrodynamic diameter, polydispersity index (PDI) and zeta-potential. Size was measured three times on the same sample, unless otherwise stated, and were reported as nr. weighted medians based on normal distributions. Zeta-potentials were measured by running 20 runs of 10 scans on one sample, unless otherwise specified. The concentration of micelle material in dispersion was determined by measuring absorption of UV light at $320 \mathrm{~nm}$ in non-crosslinked dispersions. This value was compared to the absorption of dispersions that had been subsequently lyophilized to determine the amount of micelle material.

\subsection{Formulation of ${ }^{64} \mathrm{CuCl}_{2}$ for control experiment}

To a vial containing dried ${ }^{64} \mathrm{CuCl}_{2}$ was added a solution of $\mathrm{NH}_{4} \mathrm{OAc}$ in saline $(2.3 \mathrm{~mL}$, $\mathrm{NH}_{4} \mathrm{OAc}: 10 \mathrm{mM}, \mathrm{NaCl}: 150 \mathrm{mM}, \mathrm{pH}=6.4$ ). The mixture was magnetically stirred for 30 minutes at $55^{\circ} \mathrm{C}$ and used for injection after cooling to room temperature. 


\subsection{In vivo PET/CT studies}

All animal experiments were approved by the Danish Animal Welfare Council, Ministry of Environment \& Food. Seven weeks old female NMRI nude mice purchased from Tarconic (Borup, Denmark) were inoculated in the right and left flank with $5 * 10^{6}$ U87MG cells (LGC standards, Boras, Sweden) in a 1:1 mixture with matrixgel ${ }^{\mathrm{TM}}$ (BD Biosciences, Albertslund, Denmark). Tumors were allowed to grow for 2 weeks giving tumor sizes of $133 \pm 79.9 \mathrm{~mm}^{3}$ $($ mean $\pm S D)$. The mice were divided into three groups; CL $(n=6)$ nonCL $(n=5)$ and free ${ }^{64} \mathrm{Cu}(\mathrm{n}=5)$. The $\mathrm{CL}$ group has previously been published. ${ }^{22}$ Either of the three solutions were intravenously injected in a lateral tail vein of anesthetized mice at an average dose of $7.8 \pm 0.46 \mathrm{MBq}$ (mean $\pm \mathrm{SD}$ ) for the CL micelles, $8.9 \pm 0.35 \mathrm{MBq}$ for the nonCL-micelles and $10.5 \pm 0.91 \mathrm{MBq}$ for the free ${ }^{64} \mathrm{Cu}$ at a volume of $200 \mu \mathrm{L}$. The PET/CT scans were acquired on dedicated small animal systems (all PET acquisitions on the MicroPET Focus 120, and CT acquisitions on either the MicroCAT® II or the Inveon PET/CT system, Siemens Medical Solutions, Malvern, PA, USA) at 1h, 22h and 46h post injection (p.i.) with PET acquisition times of $10,15 \& 30 \mathrm{~min}$. respectively. PET data were reconstructed with the 2-dimentional ordered-subset expectation maximization (OSEM2D) reconstruction algorithm. PET and CT images were analyzed as fused images using the Inveon software (Siemens) where regions of interest (ROIs) were drawn around liver, kidney, spleen, muscle, tumors and the left-ventricle. Uptake in the left ventricle of the heart was taken as a measure of the blood concentration. CT settings were a tube voltage of $64 \mathrm{kVp}$, a tube current of 500 $\mu \mathrm{A}, 360$ rotation steps, an exposure time of $440 \mathrm{~ms}$ and a voxel size of $0.092 \mathrm{~mm}$ on the MicroCAT® II system and a tube voltage of $65 \mathrm{kVp}$, a tube current of $500 \mu \mathrm{A}, 181$ rotation steps, an exposure time of $400 \mathrm{~ms}$ and a voxel size of $0.105 \mathrm{~mm}$ on the Inveon PET/CT system. Immediately following the last PET scan, mice were euthanized and blood as well as organs of interest collected and counted in a gamma counter (Perkin Elmer Life Sciences). The statistics were calculated using Excel 2010. Differences between groups were analyzed using an unpaired, two-tailed t-test.. A p-value $<0.05$ was considered significant. Error bars on tracer accumulation (\% ID/g) are presented as standard deviations (SD).

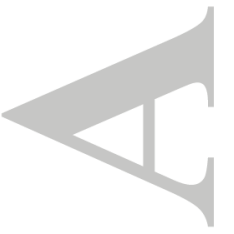




\section{Results \& discussion}

\subsection{Preparation of radiolabeled micelles}

As previously described, about 5\% of the PEG-PHEMA-PCMA monomers were conjugated with CB-TE2A. ${ }^{22}$ From these monomers, micelles were formed by dialysis. One single batch was prepared for in vivo studies, and this was split into two parts, where one was crosslinked by UV irradiation. For this reason, the only difference between the crosslinked (CL) and noncrosslinked (nonCL) micelles, apart from slight differences in radiolabeling yield, would be the crosslinking. Successful crosslinking of the CL micelles was tested by measuring the drop in UV-absorbance at $320 \mathrm{~nm}$, giving a degree of crosslinking (\%CL) of 45\% (Figure 2). In addition, crosslinking was verified by mixing the dispersions with dimethylformamide (DMF) in a micelle dispersion-to-DMF ratio of 1:16. Crosslinked micelles were found to be stable in DMF, exhibiting unchanged sizes $(34 \pm 4 \mathrm{~nm})$ and PDI (0.059), while nonCL micelles were undetectable after mixing with DMF, with poorly defined sizes and PDIs and very low count rates comparable to background $(<10)$. Crosslinking was carried out at low temperature $\left(5-7{ }^{\circ} \mathrm{C}\right)$ as we had observed that the elevated temperatures associated with irradiation could cause aggregation of certain micelle types. The CL and nonCL micelles were measured by DLS, giving hydrodynamic diameters for CL of $36 \pm 2 \mathrm{~nm}$ (PDI: $0.04 \pm$ 0.01 ) and for nonCL of $36 \pm 3 \mathrm{~nm}$ (PDI: $0.04 \pm 0.01$ ), and zeta potentials of $-3.5 \pm 0.3 \mathrm{mV}$ (CL) and -3.8 $\pm 0.4 \mathrm{mV}$ (nonCL). Accordingly, crosslinking was not observed to have significant influence on the physicochemical properties of the PMs.

The $\mathrm{CL}$ and nonCL micelles were radiolabeled with ${ }^{64} \mathrm{Cu}$ under identical circumstances with heating to $80{ }^{\circ} \mathrm{C}$ for 3 hours. Higher temperatures $\left(95{ }^{\circ} \mathrm{C}\right)$ had been observed to cause aggregation of the micelles, and since $80{ }^{\circ} \mathrm{C}$ has previously been reported for ${ }^{64} \mathrm{Cu}$-labeling of CB-TE2A this temperature was used. ${ }^{19}$ After incubation with ${ }^{64} \mathrm{Cu}$, unincorporated radioactivity was scavenged by adding EDTA. EDTA $(\operatorname{logK}: 18.5)$ is a relatively weak chelator of $\mathrm{Cu}^{2+}$, but takes it up efficiently at room temperature. Scavenging is necessary as free ${ }^{64} \mathrm{Cu}$ is known to accumulate in both tumors and liver, ${ }^{25}$ which are also intrinsic properties of nanoparticles. ${ }^{22,26}$ We had previously incubated PEG-PHEMA-PCMA monomers with no $\mathrm{CB}$-TE2A with ${ }^{64} \mathrm{Cu}$ for 3 hours at $80{ }^{\circ} \mathrm{C}$ and found that $17 \%$ of the radioactivity was associated with the micelles without EDTA scavenging, whereas 8\% was associated after EDTA scavenging. It can be assumed that this number is lower with CB- 
TE2A conjugated micelles, as a large amount of the ${ }^{64} \mathrm{Cu}$ will be taken up by the chelator. After EDTA scavenging, the radiolabeling efficiencies were, CL: $47 \%$ and NonCl: $40 \%$. The micelle material concentrations upon injection were around $1.0 \mathrm{mg} / \mathrm{mL}$ for both micelle types with corresponding specific radioactivities (at injection) of 40-45 MBq/mg.

\subsection{In vivo PET/CT studies}

Both $\mathrm{CL}$ and nonCL versions of the ${ }^{64} \mathrm{Cu}$ radiolabeled micelles were injected into the tailveins of tumor-bearing mice, followed by in vivo monitoring using PET/CT (Figure 3). Organ specific accumulation was quantified (Figure 4). Blood concentrations for both micelle types showed relatively long circulation times, with circulation half-lives on the order of 20 hours. Accordingly, the nonCL micelles were also long-circulating. At the $1 \mathrm{~h}$ time point (CL: $21.7 \pm 4.2 \% \mathrm{ID} / \mathrm{g}$, nonCL: $18.4 \pm 1.2 \% \mathrm{ID} / \mathrm{g}$ ), no statistically significant difference between the blood concentrations of $\mathrm{CL}$ and nonCL was observed $(\mathrm{p}=0.116)$, but the tendency for lower blood concentration for nonCL became significant at $22 \mathrm{~h}(\mathrm{p}=0.016)$ and $46 \mathrm{~h}(\mathrm{p}=0.004)$. However, this difference was not supported by the ex vivo data measured at $48 \mathrm{~h}$ after injection (Figure 5), where no significant difference was observed between the blood concentrations of CL and nonCL (CL: $4.2 \pm 0.8 \% \mathrm{ID} / \mathrm{g}$, nonCL: $4.0 \pm 0.4 \% \mathrm{ID} / \mathrm{g}, \mathrm{p}=$ 0.464). That a significant difference was observed only in the PET/CT images may be a consequence of the small size of the left ventricle, where partial volume effects could have influenced the PET readings. Consequently, it is reasonable to conclude that the two PM types followed similar courses of elimination from blood, with minor differences. Looking at the remaining organs, no significant differences between CL and nonCL were observed for liver, while all three measurement times showed significantly higher accumulation in spleen for $\mathrm{CL}$ compared to nonCL ( $\mathrm{p}<0.001$ ). In the kidneys, only $\mathrm{t}=22 \mathrm{~h}$ was significantly different $(\mathrm{p}=0.020)$, while muscle and tumor had $\mathrm{p}$-values above 0.05 for all measuring times. A similar picture was observed in the ex vivo data, where only tumor $(\mathrm{p}=0.023)$ and spleen $(\mathrm{p}=0.005)$ showed significant difference. Accordingly, our data suggests limited differences between the biodistributions of CL and nonCL, indicating that no pronounced advantage is obtained from crosslinking this type of PM. Although the data may hint at a marginally higher renal clearance for the nonCL PMs, this seems to hold little practical relevance for circulation times and tumor accumulation. The high stability of the nonCL 
micelles may be related to the coumarin moieties forming glassy cores with high kinetic stability.

In order to ensure that the observed similarity between the two micelle types was not simply a consequence of loss of ${ }^{64} \mathrm{Cu}$ from the PMs, control experiments using ${ }^{64} \mathrm{Cu}$ were carried out. ${ }^{64} \mathrm{CuCl}_{2}$ was injected as a non-buffered saline solution $(\mathrm{pH}=6.5)$ in which the ${ }^{64} \mathrm{Cu}$ was stabilized in solution by ammonium acetate, in order to limit the formation of ${ }^{64} \mathrm{Cu}$-containing nanocolloids. Pronounced differences between the biodistributions of the free ${ }^{64} \mathrm{Cu}$ and the PMs were observed. From the PET data (Figure 4) a fast elimination of the ${ }^{64} \mathrm{Cu}$ from the circulation was observed $(1 \mathrm{~h}=3.0 \pm 1.0 \% \mathrm{ID} / \mathrm{g})$, followed by a plateau concentration around 2-3 \% ID/g. This indicated that the ${ }^{64} \mathrm{Cu}$ was quickly taken up by the organs, followed by serum trafficking on copper-carrying proteins, notably albumin and ceruloplasmin. ${ }^{27}$ As mentioned above, significantly slower elimination of the PMs from the circulation was observed. The primary accumulation organ for $\mathrm{Cu}$ is the liver, ${ }^{27,28}$ which was also observed for free ${ }^{64} \mathrm{Cu}$ in our data, at a level that was significantly greater than for the PMs $(1 \mathrm{~h}=15.7$ $\pm 1.2 \% \mathrm{ID} / \mathrm{g}$ ). That the ${ }^{64} \mathrm{Cu}$ associated with the PMs was not cleared as rapidly and did not exhibit such high liver accumulation, supports that the ${ }^{64} \mathrm{Cu}$ was bound to the micelles in both cases. The initial accumulation of the free ${ }^{64} \mathrm{Cu}$ was followed by redistribution/elimination from the liver resulting in a drop in liver values that was faster than for the PMs. Splenic accumulation was limited for the free ${ }^{64} \mathrm{Cu}$ and significantly lower than for the PMs. Since substantial splenic accumulation of nanoparticles is routinely observed, ${ }^{29}$ this observation also supports that the ${ }^{64} \mathrm{Cu}$ was bound to the PMs. Interestingly, free ${ }^{64} \mathrm{Cu}$ showed kidney accumulation similar to the PMs. ${ }^{64} \mathrm{Cu}$ has previously been observed to accumulate in kidneys. ${ }^{25}$ Further, ${ }^{64} \mathrm{Cu}$ has also been reported to independently accumulate in tumors, ${ }^{25}$ possibly since $\mathrm{Cu}$ plays a role in tumor angiogenesis, ${ }^{30}$ which we also observed in this study. The tumor accumulation of free ${ }^{64} \mathrm{Cu}$ was comparable to the micelles, but in contrast to the PMs which showed a steady accumulation, the ${ }^{64} \mathrm{Cu}$ showed high initial accumulation $(1 \mathrm{~h}=$ $4.4 \pm 1.8 \% \mathrm{ID} / \mathrm{g}$ ), followed by a slight, though statistically insignificant, drop in values.

In summary, it appears that the nonCL micelles exhibit excellent stability in circulation, in spite of the lack of crosslinking, resulting in circulation times that are on par with the crosslinked variant. It is often assumed that crosslinking improves the pharmacokinetic profile of polymeric micelles by making them longer circulating and thereby enhancing tumor accumulation by the EPR-effect. In this study, we showed that this is not always the case in an in vivo setting, at least for 4-methylcoumarin based systems. It should be noted that 
the unimers employed here only contained 18 repeats of the 4-methylcoumarin unit and the stabilizing lipid domain was thus relatively short. We also demonstrated the usefulness of PET imaging when investigating the in vivo effect of modifications to polymeric micelles, such as crosslinking. The results presented here are interesting since the presence of single unimers without crosslinking is often preferable to cross-linked micelles, provided that the micelles exhibit sufficient kinetic stability in circulation. As the threshold for renal clearance is $<10 \mathrm{~nm},{ }^{31}$ single unimers are more easily cleared than crosslinked micelles, requiring only disintegration, rather than chemical degradation. Although coumarin-containing micelles can be de-crosslinked by light exposure, UV light cannot reach deep tissues, and is therefore generally not useful for micelle de-crosslinking after organ accumulation in the clinical setting. ${ }^{32}$ Slow clearance of therapeutically administered crosslinked polymeric micelles can potentially result in accumulation of nanoparticulate material with so far poorly understood consequences. In addition, much recent methodology is based on disintegration of micelles as a response to $\mathrm{pH}$ changes. ${ }^{33}$ Such methods can only be used if the unimers are held together by relatively weak hydrophobic interactions and the breaking of chemicals bonds from crosslinking is not required. Finally, we observed that the crosslinking step can confer some instability to the PMs if not carefully controlled, making it preferable to totally omit this step, especially when scaling up the manufacturing process.

\section{Conclusions}

In this study we compared the in vivo biodistribution of non-crosslinked polymeric micelles containing a core consisting of 18 repeats of 4-methylcoumarin with their crosslinked counterparts. The biodistributions of the two micelle types showed limited differences that can be considered negligible in a nanomedicine context. This was demonstrated against control injections of free ${ }^{64} \mathrm{Cu}$, which showed substantial tumor accumulation but differed markedly from the micelles in key organs, notably liver and spleen, as well as bloodstream concentrations. These findings are significant in that omitting crosslinking is often preferable in drug delivery due to easier clearance, less cumbersome preparation and more freedom in release strategies. Further, the demonstrated methodology showcases a general nuclear imaging technique that can be utilized for in vivo investigation of micelle stability for a range of PM types. 


\section{Acknowledgements}

This work was supported by the Technical University of Denmark (DTU), The Danish Council for Independent Research (TB; 1333-00235A), The Rigshospitalet Research Council (TB) and The Danish Cancer Society (TB; R71-A4285-13-S9).

\section{Conflicts of interest}

The authors declare no conflicts of interest.

\section{REFERENCES}

1. Talelli M, Barz M, Rijcken CJF, et al. Core-crosslinked polymeric micelles: Principles preparation, biomedical applications and clinical translation. Nano Today 2015;10:93-117.

2. Jhaveri AM, Vladimir P. Multifunctional polymeric micelles for delivery of drugs and siRNA. Front Pharmacol 2014;5:1-26.

3. Crielaard BJ, Rijcken CJF, Quan L, et al. Glucocorticoid-loaded core-cross-linked polymeric micelles with tailorable release kinetics for targeted therapy of rheumatoid arthritis. Angew Chem Int Ed 2012;51:7254 -7258.

4. Wang H, Tang L, Tu C Redox-responsive, core-cross-linked micelles capable of on-demand, concurrent drug release and structure disassembly. Biomacromolecules 2013;14:3706-3712.

5. Bontha S, Kabanov AV, Bronich TK. Polymer micelles with cross-linked ionic cores for delivery of anticancer drugs. J Control Release 2006;114:163-74.

6. Iijima M, Nagasaki Y, Okada T, et al. Core-polymerized reactive micelles from heterotelechelic amphiphilic block copolymers. Macromolecules 1999;32:1140-46.

7. Shuai X, Merdan T, Schaper AK, et al. Core-cross-linked polymeric micelles as paclitaxel carriers. Bioconjugate Chem 2004;15:441-448.

8. Ji-heung K, Emoto K, Lijima M, et al. Core-stabilized polymeric micelle as potential drug carrier: Increased solubilization of taxol. Polym Adv Technol 1999;654:647-54.

9. Rheingans O, Hugenberg N, Harris JR, et al. Nanoparticles built of cross-linked heterotelechelic, amphiphilic poly(dimethylsiloxane)-b-poly(ethylene oxide) diblock copolymers. Macromolecules 2000;33:4780-4790.

10. Liu S, Weaver JVM, Tang Y, et al. Synthesis of shell cross-linked micelles with phresponsive cores using abc triblock copolymers. Macromolecules 2002;35:6121-6131. 
11. Zhang K, Fang H, Wang Z. Cationic shell-crosslinked knedel-like nanoparticles for highly efficient gene and oligonucleotide transfection of mammalian cells. Biomaterials 2009;30:968-977.

12. Li Y, Lokitz BS, Armes SP. Synthesis of reversible shell cross-linked micelles for controlled release of bioactive agents. Macromolecules 2006;39:2726-2728.

13. Xu X, Flores JD, Mccormick CL .Reversible imine shell cross-linked micelles from aqueous raft- synthesized thermoresponsive triblock copolymers as potential nanocarriers for 'phtriggered' drug Release. Macromolecules 2011;44:1327-1334.

14. Jiang J, Qi B, Lepage M. Polymer micelles stabilization on demand through reversible photocross-linking. Macromolecules 2007;40:790-792.

15. Trenor SR, Shultz AR, Love BJ, Long TE. Coumarins in Polymers: From light harvesting to photo-cross-linkable tissue scaffolds. Chem Rev 2004;104:3059-3077.

16. He J, Zhao Y. Dyes and pigments light-responsive polymer micelles, nano- and microgels based on the reversible photodimerization of coumarin. Dyes and Pigments 2011;89:278-83.

17. Chen Y, Chou C. Reversible photodimerization of coumarin derivatives dispersed in poly(vinyl acetate). J Polym Sci A Polym Chem 1995;33:2705-2714.

18. Jin Q, Liu X, Liu G, et al. Fabrication of core or shell reversibly photo cross-linked micelles and nanogels from double responsive water-soluble block copolymers. Polymer 2010;51:1311-19.

19. Seo JW, Mahakian LM, Kheirolomoom A, et al. Liposomal Cu-64 labeling method using bifunctional chelators: poly(ethylene glycol) spacer and chelator effects. Bioconjugate Chem 2010;21:1206-15.

20. Boswell CA, Sun X, Niu W, et al. Comparative in vivo stability of copper-64-labeled crossbridged and conventional tetraazamacrocyclic complexes. J Med Chem 2004;47:1465-1474.

21. Sun X, Wuest M, Weisman GR, et al. Radiolabeling and in vivo behavior of copper-64labeled cross-bridged cyclam ligands. J Med Chem 2002;45:469-477.

22. Jensen AI, Binderup T, Kumar PEK, et al. Positron emission tomography based analysis of long-circulating cross-linked triblock polymeric micelles in a U87MG mouse xenograft model and comparison of dota and cb-te2a as chelators of copper-64. Biomacromolecules 2014; $15: 1625-33$.

23. Kumar, PEK, Almdal K, Andresen TL. Synthesis and characterization of ratiometric nanosensors for ph quantification: A mixed micelle approach. Chem Commun 2012;48:4776-4778.

24. Kumar PEK, Feldborg LN, Andresen TL. Synthesis and characterization of a micelle-based 
ph nanosensor with an unprecedented broad measurement range. Chem Mater 2013;25:1496-1501.

25. Jørgensen JT, Persson M, Madsen J, Kjær A. High tumor uptake of (64)Cu: Implications for molecular imaging of tumor characteristics with copper-based PET tracers. Nucl med biol 2013;40:345-50.

26. Frellsen AF, Hansen AE, Jølck RI, et al. Mouse positron emission tomography study of the biodistribution of gold nanoparticles with different surface coatings using embedded copper64. ACS Nano 2016;10:9887-9898.

27. Kodama H, Fujisawa C. Copper metabolism and inherited copper transport disorders: Molecular mechanisms, screening, and treatment. Metallomics 2009;1:42-52.

28. Linder MC, Hazegh-Azam M. Copper biochemistry and molecular biology. Am J Clin Nutr 1996;63:797S-811S

29. Moghimi SM, Hunter AC, Andresen TL. Factors controlling nanoparticle pharmacokinetics : an integrated analysis and perspective. Annu Rev Pharmacol Toxicol 2012;52:481-503.

30. Krajčiová D, Melník M, Havránek E, Forgácsová A. Copper compounds in nuclear medicine and oncology. Journal of Coordination Chemistry 2014;67:1493-1519.

31. Choi HS, Liu W, Misra P, et al. Renal clearance of quantum dots. Nat Biotechnol $2007 ; 25: 1165-70$.

32. Bansal A, Zhang Y. Photocontrolled nanoparticle delivery systems for biomedical applications. Acc Chem Res 2014;47:3052-3060.

33. Liu Y, Wang W, Yang J, Zhou C, Sun J. pH-sensitive polymeric micelles triggered drug release for extracellular and intracellular drug targeting delivery. Asian Journal of Pharmaceutical Sciences 2013;8:159-67. 


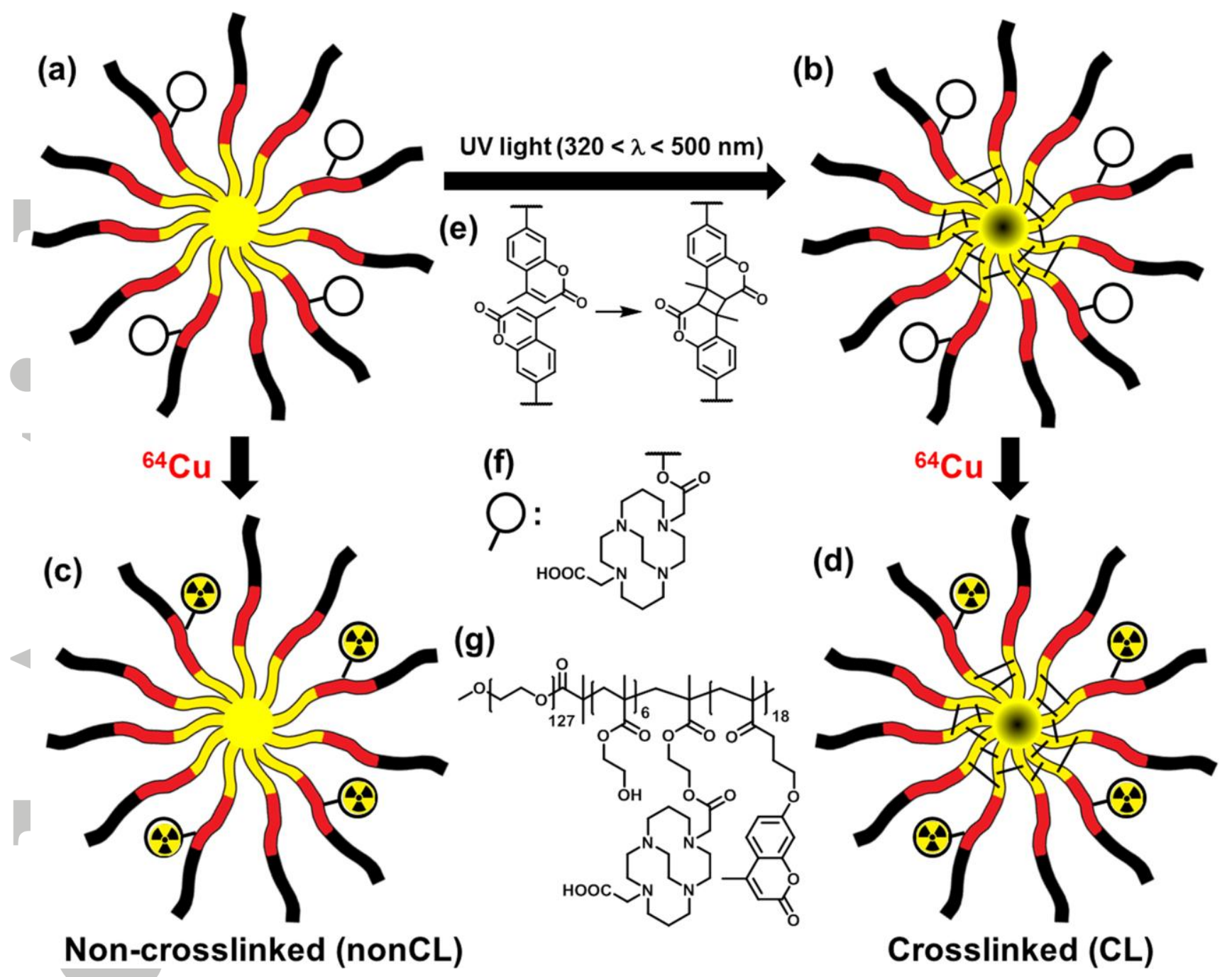

Figure 1. Concept drawing of crosslinking and composition of polymeric micelles. The triblock PEG-PHEMA-PCMA micelle unimers are shown in three colors, with yellow representing the hydrophobic core (PCMA), red the handle domain with CB-TE2A attached (PHEMA) and black the outer hydrophilic PEG domain. (a) Non-crosslinked, nonradiolabeled micelle, (b) Crosslinked, non-radiolabeled micelle, (c) Non-crosslinked micelle, radiolabeled with ${ }^{64} \mathrm{Cu}$ (nonCL), (d) Crosslinked micelle, radiolabeled with ${ }^{64} \mathrm{Cu}$ (CL), (e) Photo-induced crosslinking reaction between two 4-methylcoumarin moieties, (f) Structure of the CB-TE2A chelator, (g) Structure of the PEG-PHEMA-PCMA unimer, consisting of 18 coumarin repeats, 7 functionalizable alcohol repeats with one conjugated CB-TE2A, and 127 PEG repeats.

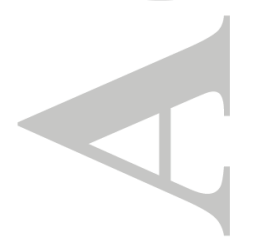




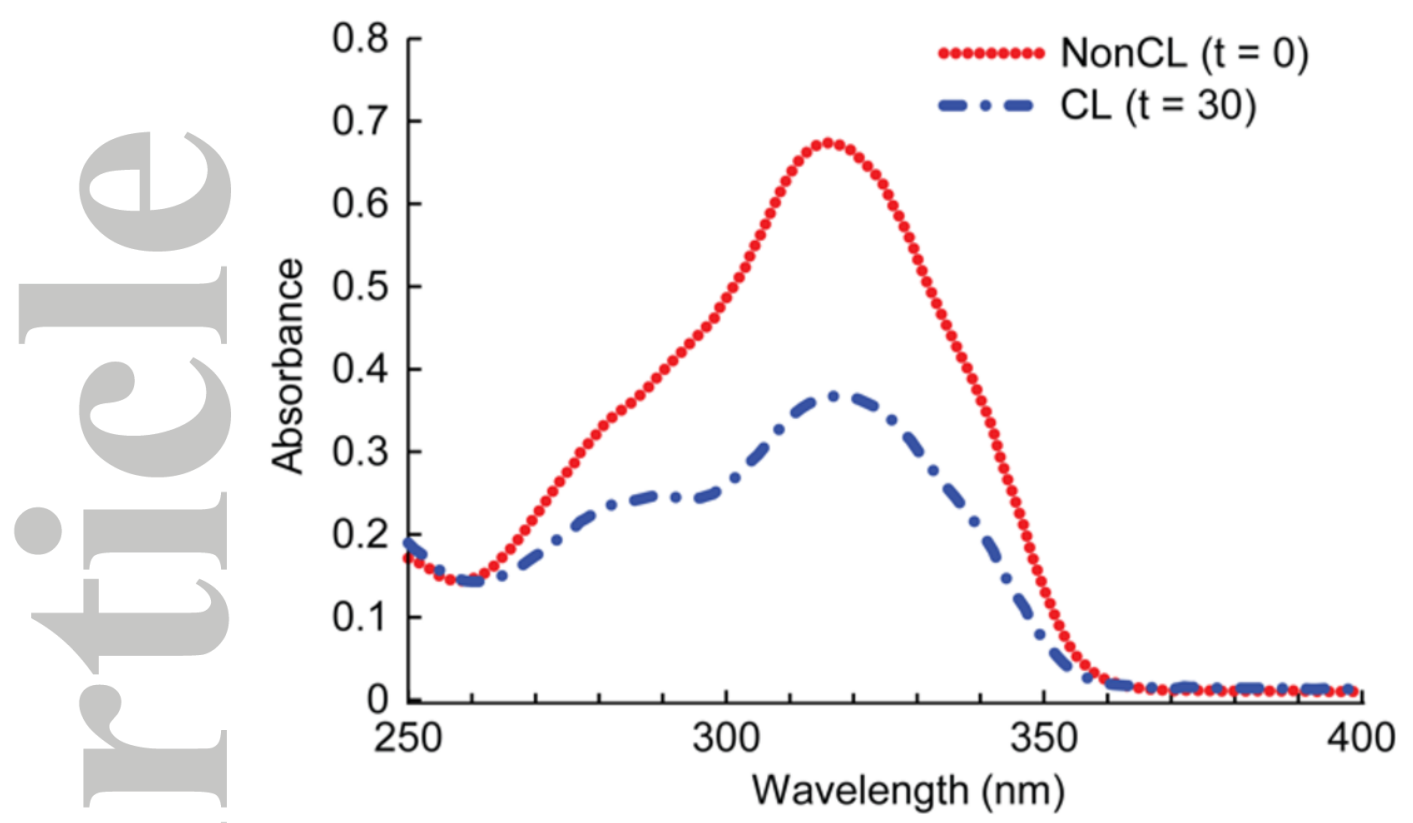

Figure 2. UV chromatograms of polymeric micelles before (NonCL) and after (CL) crosslinking for 30 minutes at $2 \mathrm{~W} / \mathrm{cm}^{2}(320-500 \mathrm{~nm})$. The degree of crosslinking was measured as the drop in absorbance of the $320 \mathrm{~nm}$ maximum from 0.664 to 0.366 giving $\% \mathrm{CL}=45 \%$.

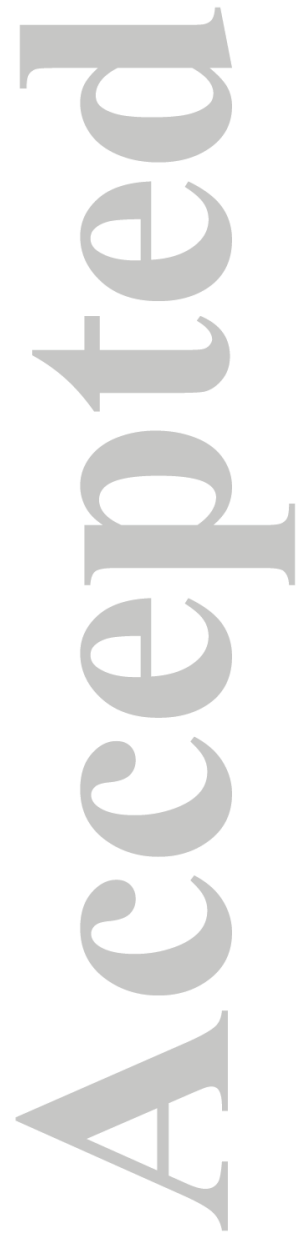




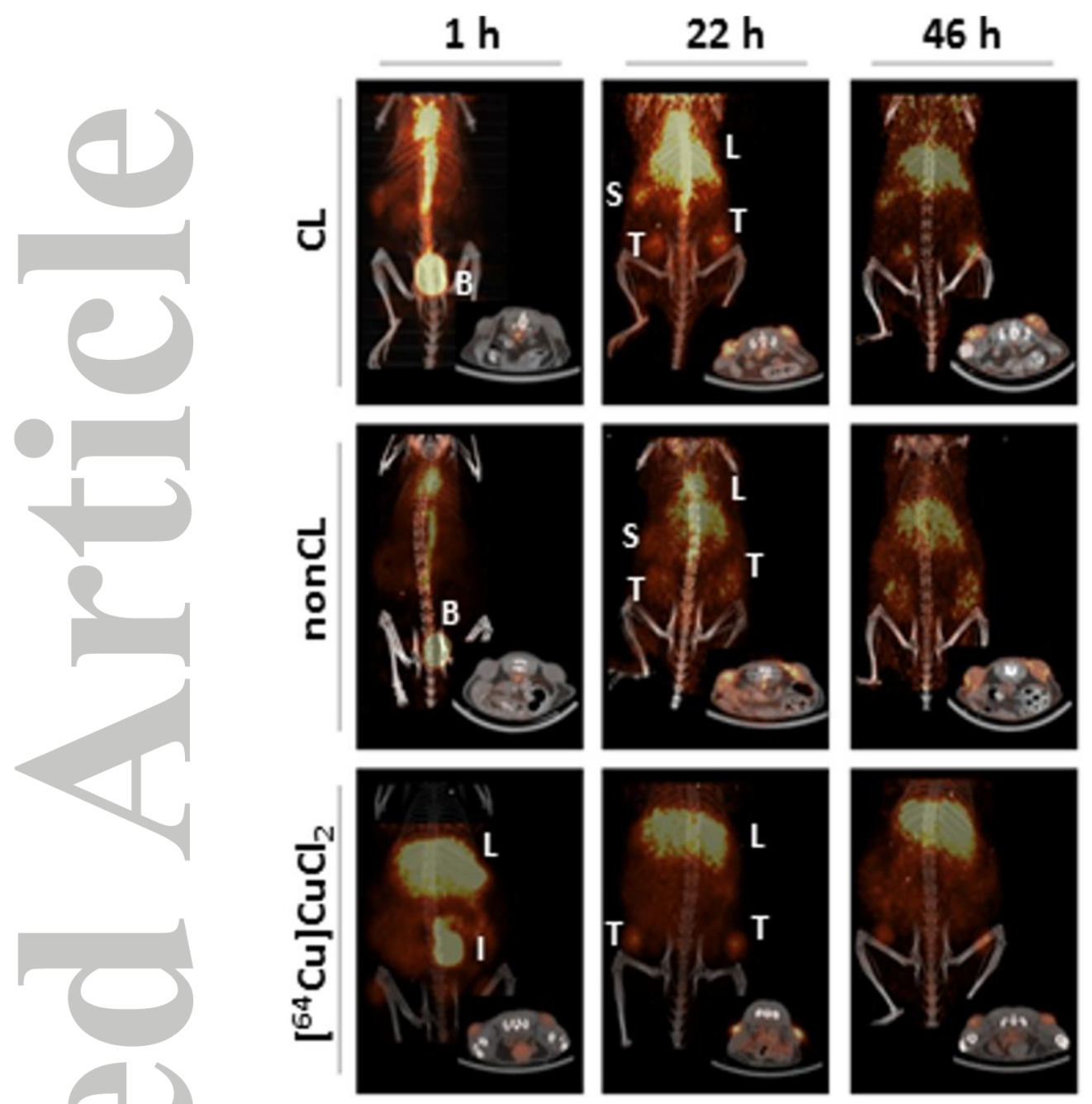

Figure 3. Fused PET/CT images of the crosslinked (CL, upper panel) and non-crosslinked (nonCL, middle panel) polymeric micelles. In the lower panel $\left({ }^{64} \mathrm{CuCl}_{2}\right)$ is shown the biodistribution of free ${ }^{64} \mathrm{Cu}$, included as control experiment. The studies were conducted in mice with U87MG tumor xenografts on both flanks. Tumors (T), liver (L), spleen (S), bladder (B) and intestine (I) are marked on the images. The images revealed gradual accumulation in liver, spleen and tumors for the two micelle types, compared to rapid $(1 \mathrm{~h})$ accumulation of the free ${ }^{64} \mathrm{Cu}$ in the liver, followed by tumor accumulation. 


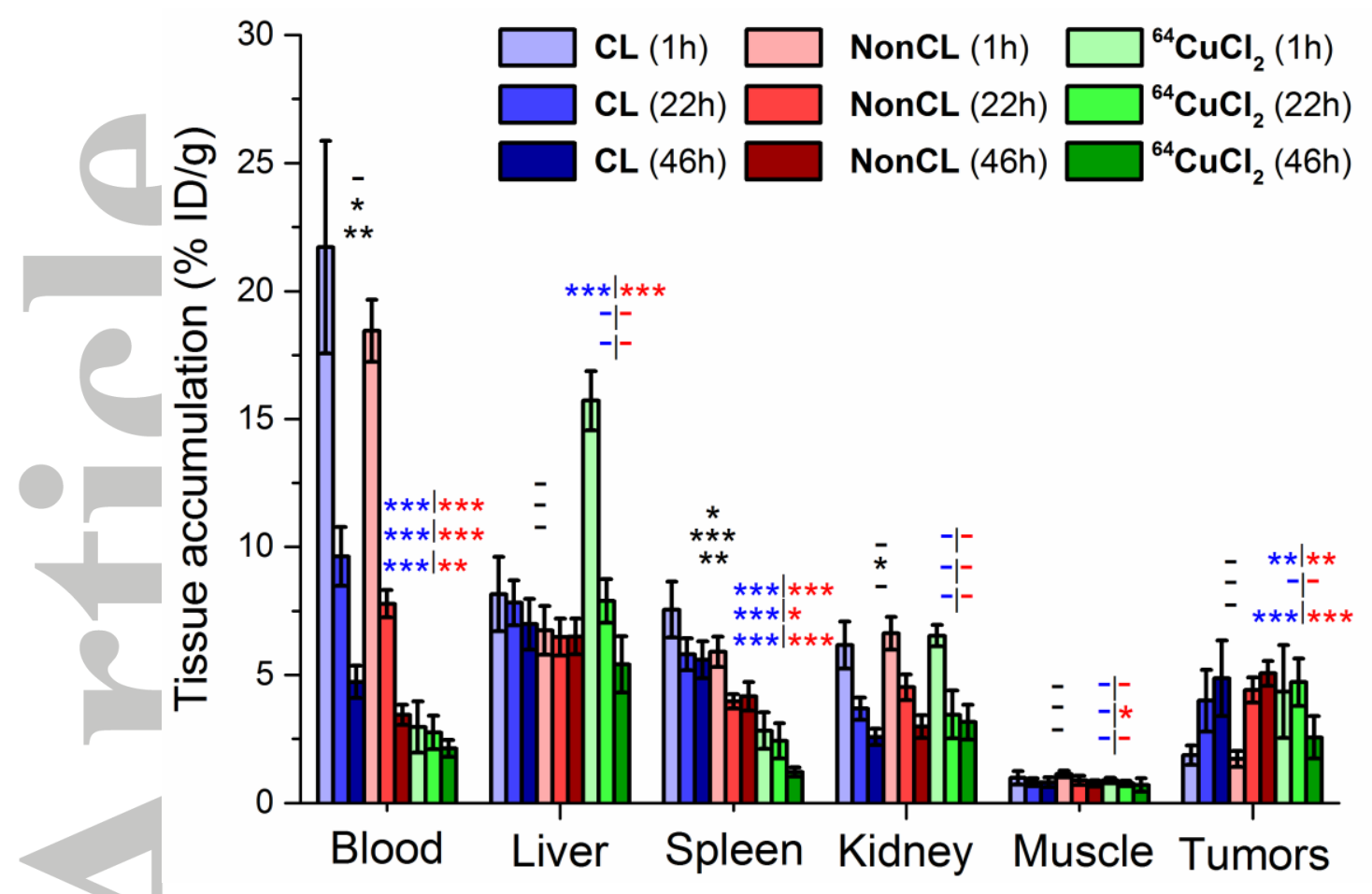

Figure 4 Mean tissue accumulation based on PET/CT imaging at 1 h, $22 \mathrm{~h}$ and $46 \mathrm{~h}$ after injection, given as \% ID/g \pm SD. Crosslinked polymeric micelles (CL) are shown in blue, noncrosslinked polymeric micelles (nonCL) is shown in red, free ${ }^{64} \mathrm{Cu}^{2+}$ is shown in green. Significant difference as per Students' T-test is shown as p >0.05 (-), $0.05>\mathrm{p}>0.01(*)$, $0.01>\mathrm{p}>0.001(* *), \mathrm{p}<0.001(* * *)$. Markings in black show $\mathrm{p}$-value comparison between $\mathrm{CL}$ and nonCL, markings in blue and red show comparison of free ${ }^{64} \mathrm{Cu}$ with $\mathrm{CL}$ or with nonCL, respectively. $\mathrm{n}=5$ for nonCL and ${ }^{64} \mathrm{CuCl}_{2}$ (tumors, $\mathrm{n}=10$ ) and $\mathrm{n}=6$ for CL (tumors, $\mathrm{n}=12)$. 


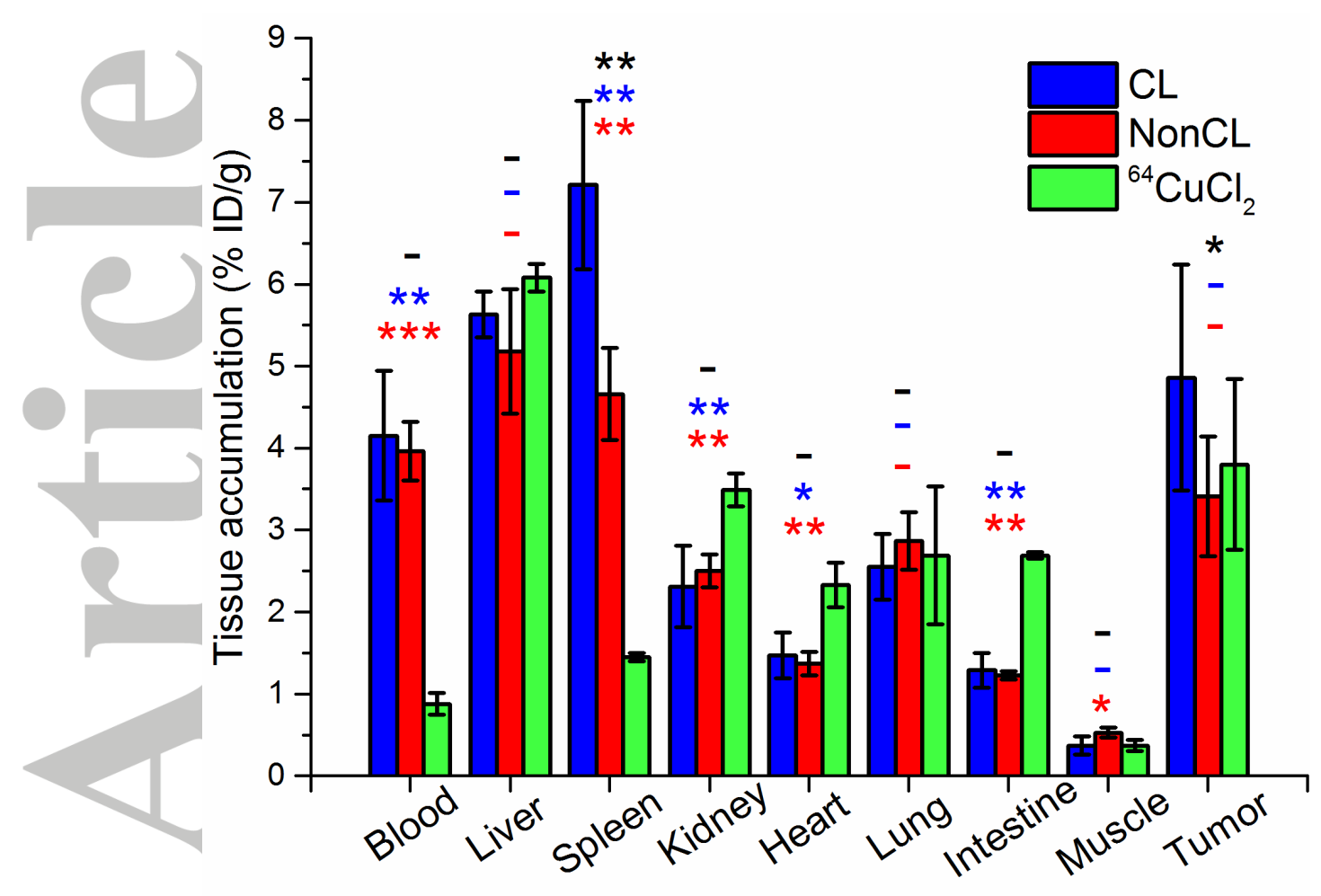

Figure 5 Mean tissue accumulation based on excised organs at $48 \mathrm{~h}$ after injection, given as $\% \mathrm{ID} / \mathrm{g} \pm \mathrm{SD}$. Crosslinked polymeric micelles (CL) are shown in blue, non-crosslinked polymeric micelles (nonCL) are shown in red, free ${ }^{64} \mathrm{Cu}^{2+}$ is shown in green. Significant difference as per Students' T-test is shown as p > $0.05(-), 0.05>\mathrm{p}>0.01(*), 0.01>\mathrm{p}>$ $0.001(* *), p<0.001(* * *)$. Markings in black show p-value comparison between CL and nonCL, markings in blue and red show comparison of free ${ }^{64} \mathrm{Cu}$ with $\mathrm{CL}$ or with nonCL, respectively. $\mathrm{n}_{\mathrm{H}}=5$ for nonCL and ${ }^{64} \mathrm{CuCl}_{2}$ (tumors, $\mathrm{n}=10$ ) and $\mathrm{n}=4$ for $\mathrm{CL}$ (tumors, $\mathrm{n}=8$ ). 